\title{
An Assessment of Risk Factors Associated with Osteoarthritis: A Study in a Tertiary Care Hospital of Bangladesh
}

\author{
Shamsul Alam ${ }^{1, ~ *, ~ E r f a n u l ~ H u q ~ S i d d i q u i ~}{ }^{2}$, Sheikh Forhad ${ }^{2}$, Anjumun Ara ${ }^{3}$, Jannat Sultana ${ }^{4}$ \\ ${ }^{1}$ Department of Orthopaedic, Gazaria Upazila Health Complex, Munshigonj, Bangladesh \\ ${ }^{2}$ Department of Orthopaedic Surgery, Bangabandhu Sheikh Mujib Medical University, Dhaka, Bangladesh \\ ${ }^{3}$ Department of Obstetrics and Gynaecology, Bangabandhu Sheikh Mujib Medical University, Dhaka, Bangladesh \\ ${ }^{4}$ Department Paediatric Medicine, Bangabandhu Sheikh Mujib Medical University, Dhaka, Bangladesh
}

\section{Email address:}

drshamsul25@yahoo (S. Alam)

${ }^{*}$ Corresponding author

\section{To cite this article:}

Shamsul Alam, Erfanul Huq Siddiqui, Sheikh Forhad, Anjumun Ara, Jannat Sultana. An Assessment of Risk Factors Associated with Osteoarthritis: A Study in a Tertiary Care Hospital of Bangladesh. Journal of Surgery. Vol. 9, No. 3, 2021, pp. 98-102. doi: $10.11648 /$ j.js.20210903.11

Received: March 20, 2021; Accepted: April 2, 2021; Published: April 29, 2021

\begin{abstract}
Introduction: Osteoarthritis (OA) is a common musculoskeletal disorder which occur in different patterns. It is the most common form of arthritis, affecting millions of people worldwide year after year. It occurs when the protective cartilage that cushions the ends of your bones wears down over time. OA can damage any joint that affects joints in one's hands, knees, hips and spine. As there had been very few studies in Bangladesh we have not enough data regarding the risk factors of osteoarthritis. Objective: The aim of this prospective observational study was to evaluate the risk factors associated with osteoarthritis (OA). Methodology: This was a retrospective study which was conducted at the Department of Orthopedics Surgery in Bangabandhu Sheikh Mujib Medical University (BSMMU), Dhaka, Bangladesh during the period from January 2018 to December 2018. Data were collected by a pre-designed questioner and analyzed by computer program, SPSS Version 20. Incomplete data-sheets were rejected before analysis and finally total study population was fixed to 146 with Osteoarthritis (OA). Result: Risk factor, family history was associated with 29 (19.86\%) patients. It was the highest engagement and it was followed by Diabetes Mellitus: 27 (18.49\%), history of injury: 14 (9.59\%), Hypertension: 12 (8.22\%) and previously operated: 7 (4.79\%). In This study the comparison of sample data regarding risk factors have been displayed. Here against ACR, LEFS, $\mathrm{K}$ and $\mathrm{L}$ and VAS score $\mathrm{P}$ values were $0.876,0.893,0.067$ and 0.436 respectively. So there was not any significant correlation. Conclusion: In this study 'family history of osteoarthritis' and 'Diabetes Mellitus (DM)' were two most frequent risk factors which was associated with osteoarthritis. But we did not find any significant correlation regarding any risk factor. More studies regarding $\mathrm{OA}$ are necessary to get the clear concepts about this issue.
\end{abstract}

Keywords: Osteoarthritis, Risk Factors, Overweight, Risk Factors, K and L Score, Cartilage

\section{Introduction}

Osteoarthritis is the most common form of arthritis, affecting millions of people worldwide year after year. It occurs when the protective cartilage that cushions the ends of your bones wears down over time. Although Osteoarthritis can damage any joint, the disorder most commonly affects joints in your hands, knees, hips and spine. Osteoarthritis
(OA) is the most common type of rheumatic disease and a leading cause of disability. More than 10 percent of the world populations have OA. As OA progresses, severe joint pain limits patients' physical activity. [1] Many of the recent studies have conducted comparisons between urban and rural areas and poor and affluent communities. [2] Joint-related pain generally triggers those with osteoarthritis to pursue medical or surgical treatment. Therefore, we need to focus on investigating the contributing factors related to joint-related 
pain, along with associated factors in osteoarthritis. Osteoarthritis is characterized by loss of articular cartilage, hypertrophy of bone at the margins, subchondral sclerosis and range of biochemical and morphological alterations of the synovial membrane and joint capsule and is caused due to chronic degeneration of the articulating surfaces of the joint. [3] Late stage changes of OA include softening, ulceration of the articular cartilage, synovial inflammation also may occur. Patients usually present with symptoms of pain, particularly after prolonged activity and weight bearing; whereas stiffness is experienced after inactivity. [4] Most patients with Osteoarthritis have no known cause. This is known as Primary osteoarthritis. Primary osteoarthritis is mostly related to aging. It can be localized, generalized or as erosive osteoarthritis. Secondary osteoarthritis is caused by another disease or condition. Osteoarthritis (OA) is a common degenerative disease of joints. The major clinical features are pain and stiffness, leading to a decline in physical function. Considerable pain and disability result from large joint OA, which is the major cause of joint replacement surgery and cost to the community. The focus of current medical intervention is on symptomatic relief, given that no cure for the disease exists. As curative intervention is unlikely in the near future, both the prevention of disease onset and progression of the disease are of great importance. OA of the knee is a common form of arthritis in the elderly. It is a complex multifactorial disease. Risk factors are broadly divisible into those that are constitutional or genetic and those that are local and driven by biomechanical elements, such as joint usage. As incidence of knee OA is expected to rise as the proportion of elderly population continues to increase and knee OA has a substantial impact on activities of daily living, several epidemiological studies in Western and Oriental countries [1-5] have investigated risk factors of knee $\mathrm{OA}$, finding a consistent association between the incidence or progression of knee OA and age, obesity, history of knee injury, occupational physical demands, physical activity, and regular sport activities.

\section{Material and Methods}

This prospective observational study was conducted at the Department of Orthopedics Surgery in Bangabandhu Sheikh Mujib Medical University (BSMMU), Dhaka, Bangladesh during the period from January 2018 to December 2018. In total 146 patients attended with osteoarthritis (Diagnosed) was the total study population. Proper written consent in language understood by the patient was taken. Personal data, clinical findings, radiological findings, biochemical reports and related medical records of all patients were obtained. A pre-tested semi structured questionnaire was used in data collection. Through the questionnaire we collected the data regarding the patient's socio-demographic details, the possible risk factors for developing OA, age, gender, occupation, family history of OA, physical activity, history of injury. X-ray, weight and height measurement along with all necessary investigations according to standard protocol were done. On basic evaluation, patients having symptoms suggestive of OA were sent for X-rays. On X-ray, Grade 2 patients were excluded from the study and Grade 0 and Grade 1 patients were taken in the study. Baseline biochemical parameters of these patients were recorded, and patients were followed up after 3 and 6 months. Simultaneously, patients were started on medication and physiotherapy. The inclusion criteria were: a) Early OA knee-Kellgren and Lawrence ( $\mathrm{K}$ and $\mathrm{L}$ ) Grade 0 and $1, \mathrm{~b}$ ) Qualitative C - reactive protein - Negative, c) Erythrocyte Sedimentation Rate $<20 \mathrm{~mm} / 1 \mathrm{hr}$, d) Serum Uric Acid level $<$ $6 \mathrm{mg} / \mathrm{dl}$, e) Total Leucocyte count $<10000 / \mathrm{Cu} . \mathrm{mm}$. On the other hand the exclusion criteria were: a) Advanced OA knee with Kellgren And Lawrence ( $\mathrm{K}$ and $\mathrm{L}$ ) $>$ Grade 2, b) Joints pain due to Rheumatological or Systemic diseases, c) Malalignment of Weight Bearing Axis and d) Internal Knee derangement causing knee pain. Statistical analysis was conducted by using SPSS Version 20 software. An unpaired $t$ test was used to test the null hypothesis. The procedure calculates the difference between the observed means in two independent samples. A significance value (P-value) and 95\% Confidence Interval (CI) of the difference is reported. The Pvalue is the probability of obtaining the observed difference between the samples if the null hypothesis were true. The null hypothesis is the hypothesis that the difference is $0 . \mathrm{P}$ value less than 0.05 is considered significant.

\section{Result}

In our study among total 146 participants 67 were male which was $46 \%$ and 79 were female which $54 \%$ of the total study population. So here female are dominating in number. In this study, the age range of our participants was from 21 to 80 years. The highest number of patients was from 51-60 years' age group. It was 52 in number which was more than one third of the total population (35.62\%). Then from 41-50 years' age group it was 37 (25.34), from 61-70 years' age group: 24 (16.44\%), from 31-40 years' age group it was 15 $(10.27 \%)$, from $>70$ years' age group it was $9(6.16 \%)$ and from 21-30 years' age group it was found another 9 (6.16\%). According to the socioeconomic status and monthly family income in this study all the responded were divided into three classes: lower class, middle class, and upper class. The 'per month family income' of the respondents of lower class, middle class, and upper class were defined as $\leq 9000.00$ BDT, 9000-20000 BDT and $\geq 20000$ BDT respectively. The highest numbers of patients of our study come from lower class. This number was 87 which were $60 \%$ of the total study population. Then it followed by 46 (31\%) from middle class and only 13 (9\%) from upper class. The highest number patient's BMI Score was 25-30 who were over weighted. This number was 77 which were $52.74 \%$. Then it followed by normal weighted, BMI <18.5-24.9: 49 (33.56\%); obese, BMI >30: 14 (9.59\%) and under weighted, BMI : <18.5: 6 (4.11\%). On evaluating relation of posture and habits with Osteoarthritis, it was found that most of the patients were associated with some posture and habits which may associate with Osteoarthritis. 
We found only $15 \%$ patients with normal posture and habits. $30 \%$ patients were engaged with stair climbing which was the highest ratio. Risk factor, family history was associated with $29(19.86 \%)$ patients. It was the highest engagement and it was followed by Diabetes Mellitus: 27 (18.49\%), history of injury: 14 (9.59\%), Hypertension: 12 (8.22\%) and previously operated: 7 (4.79\%). In This study the comparison of sample data regarding overweight have been analyzed. Here we did not get any significant correlation. In this aspect against ACR, LEFS, $\mathrm{K}$ and L and VAS score the P values were $0.944,0.989$, 0.973 and 0.522 respectively. In Table 5 the comparison of sample data regarding menstruating stages have been displayed. Here against ACR, LEFS, K and L and VAS score $P$ values were $1.000,0.624,0.595$ and 0.309 respectively. So there was not any significant correlation. In Table 6 the comparison of sample data regarding risk factors have been displayed. Here against ACR, LEFS, K and L and VAS score $P$ values were $0.876,0.893,0.067$ and 0.436 respectively. So there was not any significant correlation.

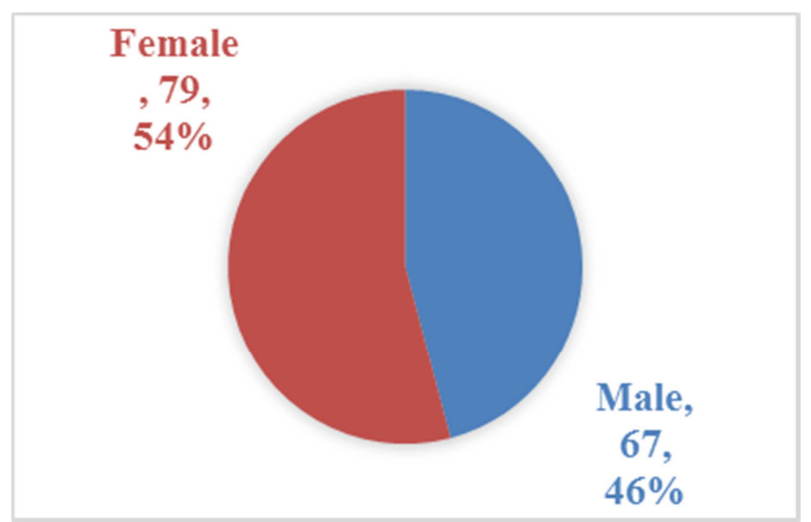

Figure 1. Male-female ratio of the participants $(n=146)$.

Table 1. Age distribution of the participants $(n=146)$.

\begin{tabular}{lll}
\hline Age Group & $\mathbf{n}$ & $\mathbf{\%}$ \\
\hline $21-30$ & 9 & 6.16 \\
$31-40$ & 15 & 10.27 \\
$41-50$ & 37 & 25.34 \\
$51-60$ & 52 & 35.62 \\
$61-70$ & 24 & 16.44 \\
$>70$ & 9 & 6.16 \\
\hline
\end{tabular}

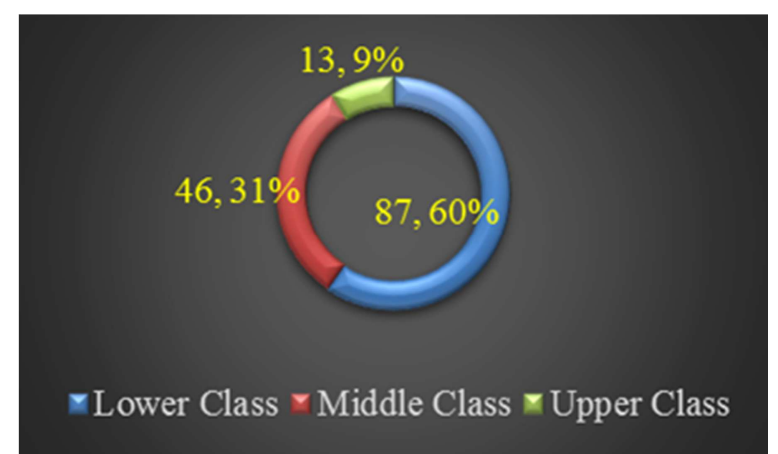

Figure 2. Family status of the participants $(n=146)$.

Table 2. Ratio of participants according to BMI Scores (n=146).

\begin{tabular}{lll}
\hline BMI Score & n & \% \\
\hline Underweight $(<18.5)$ & 6 & 4.11 \\
Normal weight $(<18.5-24.9)$ & 49 & 33.56 \\
Overweight $(25-30)$ & 77 & 52.74 \\
Obese $(>30)$ & 14 & 9.59 \\
Total & 146 & 100 \\
\hline
\end{tabular}

Table 3. Risk factors associated with osteoarthritis.

\begin{tabular}{lll}
\hline Particulars & n & \% \\
\hline Diabetes Mellitus & 27 & 18.49 \\
Hypertension & 12 & 8.22 \\
Previously operated & 7 & 4.79 \\
History of injury & 14 & 9.59 \\
Family History & 29 & 19.86 \\
\hline
\end{tabular}

Table 4. Comparison of sample data regarding overweight.

\begin{tabular}{llll}
\hline \multirow{2}{*}{ Scale } & Non Obese $\mathbf{n}=\mathbf{1 4}$ & Obese $\mathbf{n}=\mathbf{1 4}$ & \multirow{2}{*}{ P value } \\
\cline { 2 - 3 } & MEAN Difference & MEAN Difference & \\
\hline ACR & $0.811 \pm 0.706$ & $0.830 \pm 0.715$ & 0.944 \\
LEFS & $(-) 14.734 \pm 8.427$ & $(-) 14.687 \pm 9.227$ & 0.989 \\
K and L & $0.875 \pm 0.705$ & $0.866 \pm 0.695$ & 0.973 \\
VAS & $2.138 \pm 0.591$ & $1.991 \pm 0.607$ & 0.522 \\
\hline
\end{tabular}

Table 5. Comparison of sample data regarding menstruating stages in female participants.

\begin{tabular}{llll}
\hline \multirow{2}{*}{ Scale } & Menstruating $(\mathbf{n}=12)$ & Post-menopausal $(\mathbf{n}=\mathbf{1 2})$ & \multirow{2}{*}{ P value } \\
\cline { 2 - 3 } & MEAN Difference & MEAN Difference & \\
\hline ACR & $1 \pm 0.786$ & $1 \pm 0.822$ & 1.000 \\
LEFS & $(-) 18.665 \pm 12.268$ & $(-) 21.073 \pm 11.538$ & 0.624 \\
K and L & $0.812 \pm 0.768$ & $0.973 \pm 0.692$ & 0.595 \\
VAS & $2.113 \pm 0.787$ & $1.775 \pm 0.804$ & 0.309 \\
\hline
\end{tabular}

Table 6. Comparison of sample data regarding risk factors.

\begin{tabular}{llll}
\hline \multirow{2}{*}{ Scale } & With Risk Factors $\mathbf{n = 8 9}$ & Without Risk Factors n=89 \\
\cline { 2 - 3 } & MEAN Difference & MEAN Difference \\
\hline ACR & $0.903 \pm 0.811$ & $0.922 \pm 0.809$ & 0.876 \\
LEFS & $(-) 14.920 \pm 6.841$ & $(-) 14.771 \pm 7.917$ \\
K and L & $0.704 \pm 0.825$ & $0.920 \pm 0.736$ & 0.893 \\
VAS & $1.729 \pm 0.690$ & $1.812 \pm 0.728$ \\
\hline
\end{tabular}

\section{Discussion}

The aim of this prospective observational study was to evaluate the risk factors associated with osteoarthritis (OA). During the study we found some risk factors like Diabetes Mellitus, hypertension, previously operated cases, history of injury and family history. Besides those, some factors like obesity, postures age and gender also attracted our concentration. In our study an almost equal proportion of males and females was seen, which suggests that early OA changes need not be gender specific either, as supposed to the late changes, which are predominantly seen in females. [5] Socio economic factor seems to play a key role, as $60 \%$ of 
the study population belonged to the lower and $31 \%$ middle classes. This should be ought to the possibility that most population belonging to this demographic usually engage in occupations that require more physical activity. This leads to more biomechanical stress to the knee joint, and hence possibly a higher prevalence. Another contributing factor might be the lifestyle habits of people in this population, such as use of toilet, walking as a mode of transport due to lack of vehicles etc. In many studies higher BMI considered as another significant factor associated with osteoarthritis and a contributing factor to current arthritic pain at the same time. Overweight is thought to cause osteoarthritis by increasing the load exerted on the joints. [6, 7] However, it is also known to be associated with hand osteoarthritis, where being overweight does not necessarily cause increased joint load. [8, 9] Previous studies have suggested that systemic and metabolic factors associated with overweight might play a role in inflammatory processes. [10] Studies have suggested that nutrition plays a role in osteoarthritis. [11] For example, antioxidants were found to re- duce the progression of osteoarthritis, although they did not significantly reduce the initiation of osteoarthritis. [12] In addition, some nutritional factors have been found to exhibit a relationship with the inflammatory process and modulating chronic pain. [13] However, this study failed to reveal the role of nutrition intake in current arthritis pain among older adults with osteoarthritis. A more intensive and detailed study design would be needed to investigate the relationship between osteoarthritis and nutritional state. The fact that approximately $58 \%$ of the population had a BMI belonging to the overweight or obese category further cements the fact that BMI is a strong contributing risk factor to OA Knee. [14] In this study, we also aimed to investigate whether risk factors can alter the degree of improvement in patients that present with early changes of OA. Hence, ACR, LEFS K and $\mathrm{L}$ and VAS scoring of patients were compared in different groups at the beginning and end of the study. These were compared for menstruating and post-menopausal women, obese and non-obese patients, patients with daily activities considered as risk factors and those without, and patients with and without family history. Although in our study we could not concentrate on Osteoporosis but Osteoporosis was another factor associated with osteoarthritis. The relationship between Osteoarthritis and Osteoporosis has been somewhat controversial. In our study, risk factor, family history was associated with $29(19.86 \%)$ patients. It was the highest engagement and it was followed by Diabetes Mellitus: 27 (18.49\%), history of injury: 14 (9.59\%), Hypertension: 12 $(8.22 \%)$ and previously operated: 7 (4.79\%). In This study the comparison of sample data regarding overweight have been analyzed. Here we did not get any significant correlation. In this aspect against ACR, LEFS, K and L and VAS score the $\mathrm{P}$ values were $0.944,0.989,0.973$ and 0.522 respectively. In Table 5 the comparison of sample data regarding menstruating stages have been displayed. Here against ACR, LEFS, $\mathrm{K}$ and $\mathrm{L}$ and VAS score $\mathrm{P}$ values were $1.000,0.624,0.595$ and 0.309 respectively. So there was not any significant correlation. In Table 6 the comparison of sample data regarding risk factors have been displayed. Here against ACR, LEFS, $\mathrm{K}$ and $\mathrm{L}$ and VAS score $\mathrm{P}$ values were $0.876,0.893,0.067$ and 0.436 respectively. So there was not any significant correlation.

\section{Conclusion \& Recommendation}

In this study 'family history of osteoarthritis' and 'Diabetes Mellitus (DM)' were two most frequent risk factors which was associated with osteoarthritis. But we did not find any significant correlation regarding any risk factor. The findings may be helpful for farther study and in the treatment procedure of Osteoarthritis (OA). There were some limitations in our study. Basically it was a single cantered study. The sample size was also smaller. So the findings of this study may not reflect the exact scenario of the whole country. We would like to recommend for conducting more study related to Osteoarthritis for getting more clear concepts.

\section{References}

[1] Wang Y, Fransen M, Bridgett L, March L, Hoy D, Penserga E, et al. (2011) The epidemiology of osteoarthritis in Asia. Int J Rheum Dis 14: 113-121.

[2] Cooper C, Altman R, Asch E, Bloch D, Bole G, Borenstein D, et al. (1986) Development of criteria for the classification and reporting of osteoarthritis. Classification of osteoarthritis of the knee. Diagnostic and Therapeutic Criteria Committee of the American Rheumatism Association. Arthritis Rheum 29: 1039-1049.

[3] Biological markers in osteoarthritis Jean-Charles Rousseau and Pierre D Delmas.

[4] Global burden of osteoarthritis in the year 2000 Deborah Symmons1 Colin Mathers2, Bruce Pfleger3.

[5] Osteoporosis and osteoarthritis: shared mechanisms and epidemiology. Geusens PP, van den Bergh JP. Curr Opin Rheumatol. 2016 Mar; 28 (2): 97-103. doi: 10.1097/BOR.0000000000000256. Review. PMID: 26780427.

[6] Felson DT. Obesity and vocational and avocational overload of the joint as risk factors for osteoarthritis. J Rheumatol Suppl 2004; 70: 2-5.

[7] Messier SP, Gutekunst DJ, Davis C, DeVita P. Weight loss reduces knee-joint loads in overweight and obese older adults with knee osteoarthritis. Arthritis Rheum 2005; 52: 2026-32.

[8] Grotle M, Hagen KB, Natvig B, Dahl FA, Kvien TK. Obesity and osteoarthritis in knee, hip and/or hand: an epidemiological study in the general population with 10 years follow-up. BMC Muscu- loskelet Disord 2008; 9: 132.

[9] Dahaghin S, Bierma-Zeinstra SM, Koes BW, Hazes JM, Pols HA. Do metabolic factors add to the effect of overweight on hand osteo- arthritis? The Rotterdam Study. Ann Rheum Dis 2007; 66: 916-20.

[10] Wolfe F. The C-reactive protein but not erythrocyte sedimentation rate is associated with clinical severity in patients with osteoarthri- tis of the knee or hip. J Rheumatol 1997; 24: 1486-8. 
[11] Sokoloff L, Mickelsen O. Dietary fat supplements, body weight and osteoarthritis in dba-2jn mice. J Nutr 1965; 85: 117-21.

[12] McAlindon TE, Jacques P, Zhang Y, Hannan MT, Aliabadi P, Weissman B, et al. Do antioxidant micronutrients protect against the development and progression of knee osteoarthritis? Arthritis Rheum 1996; 39: 648-56.
[13] Schwartz ER, Oh WH, Leveille CR. Experimentally induced os- teoarthritis in guinea pigs: metabolic responses in articular carti- lage to developing pathology. Arthritis Rheum 1981; 24: 1345-55.

[14] Osteoarthritis, obesity and type 2 diabetes: The weight of waist circumference. Duclos M. Ann Phys Rehabil Med. 2016 Jun; 59 (3): 157-160. doi: 10.1016/j.rehab.2016.04.002. Epub 2016 May 19. 